\section{HEPATITIS A AGUDA EN PACIENTES CON SÍNDROME FEBRIL EN UN HOSPITAL DEL NORTE DEL PERÚ}

\section{ACUTE HEPATITIS A IN PATIENTS WITH FEBRILE ILLNESS IN A HOSPITAL IN THE NORTHERN REGION OF PERU}

\section{Heber Silva-Díaz'a , Tatiana D. Chávez-Orosco²,b, Katya M. Mera-Villasis ${ }^{1, c}$, Franklin R. Aguilar-Gamboa ${ }^{1, c}$, Mayra Y. Valderrama-Ayén ${ }^{1, c}$}

Sr. Editor. Hemos leído con interés el estudio publicado en el número anterior de la revista, referido a la frecuencia de hepatitis A en niños y adolescentes de cinco ciudades del Perú (1). El artículo es interesante e importante por cuanto actualiza el estado de la seroprevalencia y los patrones de endemicidad en varias regiones del Perú. Sin embargo, aún se desconoce la frecuencia actual de morbilidad asociada con hepatitis $A$ aguda, en un contexto posterior a la introducción de una vacuna efectiva. En áreas con alta endemicidad la infección es asintomática, pero en aquellas con endemicidad intermedia la morbilidad se incrementa con síntomas debilitantes y hepatitis fulminante que se asocia con una alta mortalidad ${ }^{(2)}$, razón por la cual en la presente comunicación se desea compartir los resultados de un estudio sobre la frecuencia de hepatitis A aguda en pacientes con síndrome febril admitidos un hospital del norte del Perú.

Se realizó un estudio descriptivo transversal en 259 pacientes atendidos en el Hospital Regional Lambayeque (categoría III-1), durante enero de 2014 a septiembre de 2015. Se excluyeron a niños menores de 2 años y recientemente vacunados. La hepatitis $A$ aguda se determinó mediante la detección de anticuerpos séricos IgM anti-VHA usando la técnica de ELISA (HAV IgM ELISA kit de CTK Biotech, ISA). Adicionalmente, se recolectaron datos de edad, sexo, región de procedencia, zona de residencia y tipo de agua de consumo. El estudio fue revisado y aprobado por el comité de ética del Hospital Regional Lambayeque.

La mediana de la edad fue de 30 años, rango intercuartileo de 17 a 49 . En el 6,9\% (18/259) de pacientes febriles se demostró la presencia de anticuerpos IgM anti-VHA (IC $95 \%: 1,35$ a 12,55). El análisis bivariado mostró que el grupo etario de 0 a 17 años $(p=0,001 ; O R=4,24)$ y los pacientes que procedieron de la región de Lambayeque $(p=0,026)$ estuvieron asociados con la mayor frecuencia de anticuerpos IgM anti-VHA (Tabla 1).

Tabla 1. Características de los pacientes con síndrome febril atendidos en el Hospital Regional Lambayeque, Chiclayo, 2014-2015

\begin{tabular}{|c|c|c|c|}
\hline Variables & $\mathrm{N}=259(\%)$ & IgM anti-HVA/total (\%) & Valor $p$ \\
\hline \multicolumn{4}{|l|}{ Sexo } \\
\hline Masculino & $127(49,0)$ & $10 / 127(7,9)$ & $0,630^{*}$ \\
\hline Femenino & $132(51,0)$ & $8 / 132(6,1)$ & \\
\hline \multicolumn{4}{|l|}{ Grupo etario (años) } \\
\hline 0 a 17 & $67(25,9)$ & $11 / 67(16,4)$ & $0,001^{* *}$ \\
\hline 18 a 59 & $158(61,0)$ & $7 / 158(4,43)$ & \\
\hline 60 a más & $34(13,1)$ & $0 / 34(0,0)$ & \\
\hline \multicolumn{4}{|c|}{ Región de procedencia } \\
\hline Amazonas & $19(7,3)$ & $0 / 19(0,0)$ & $0,026^{* *}$ \\
\hline Cajamarca & $48(18,5)$ & $0 / 48(0,0)$ & \\
\hline Lambayeque & $175(67,6)$ & $18 / 175(10,3)$ & \\
\hline Otro & $17(6,6)$ & $0 / 17(0,0)$ & \\
\hline \multicolumn{4}{|l|}{ Zona de residencia } \\
\hline Rural & $117(45,2)$ & $6 / 117(5,1)$ & $0,295^{*}$ \\
\hline Urbano & $142(54,8)$ & $12 / 142(8,5)$ & \\
\hline \multicolumn{4}{|c|}{ Agua de consumo en casa } \\
\hline No potable & $95(36,7)$ & $9 / 95(9,5)$ & $0,224^{*}$ \\
\hline Potable & $164(63,3)$ & $9 / 164(5,5)$ & \\
\hline
\end{tabular}

* Fisher exacta; ** chi cuadrado

Laboratorio de Investigación, Hospital Regional Lambayeque. Lambayeque, Perú.

2 Facultad de Ciencias de la Salud, Universidad Particular de Chiclayo. Lambayeque, Perú.

a Biólogo microbiólogo, doctor en ciencias; ${ }^{\mathrm{b}}$ tecnólogo médico; ${ }^{\mathrm{c}}$ biólogo microbiólogo

Recibido: 29-11-15 Aprobado: 16-12-15

Citar como: Silva-Díaz H, Chávez-Orosco TD, Mera-Villasis KM, Aguilar-Gamboa FR, Valderrama-Ayén MY. Hepatitis a aguda en pacientes con síndrome febril en un hospital del norte del Perú [carta]. Rev Peru Med Exp Salud Publica. 2016;33(1):177-8. doi: 10.17843/rpmesp.2016.331.1887 
La mayor frecuencia en el grupo de 0 a 17 , con un promedio de edad de 11 años en los positivos, confirma el incremento de edad en la infección en los pacientes estudiados ${ }^{(1)}$. No obstante, también se observó que el $4,4 \%(7 / 158)$ de pacientes entre 18 a 59 años tuvieron hepatitis $A$ aguda, lo que epidemiológicamente es importante, debido a que los casos severos son más probables cuando la infección ocurre a mayor edad (3). En las dos últimas décadas, debido al renuente interés, se ha visto limitado el progreso del conocimiento sobre la patogénesis e inmunidad contra el VHA; aun cuando el promedio de edad de la infección se ha incrementado en países en desarrollo como Perú, significando una mayor cantidad de hepatitis severa cuyos mecanismos son pobremente conocidos ${ }^{(3,4)}$.

Por otro lado, el hallazgo de que todos los casos positivos procedieran de la región Lambayeque, se puede explicar por la mayor proporción de pacientes en el estudio $(67,6 \%)$, y por el probable nivel de endemicidad menor, en comparación a las otras regiones (Cajamarca y Amazonas), lo que estaría condicionando una mayor morbilidad. Cabe resaltar que el sexo, la zona de residencia rural o urbana y el tipo de agua de consumo no representaron asociación con la mayor frecuencia de anticuerpos IgM contra la enfermedad, probablemente por la existencia de múltiples factores de riesgo y fuentes de infección comunes.

Se enfatiza la necesidad mayores estudios que evalúen la incidencia actual de la hepatitis A aguda en el Perú, y su variación tras la introducción de la vacuna contra la infección; así como lo muestra un estudio en Estados Unidos, donde desde el 1995 al 2007 la hepatitis A aguda ha disminuido en $92 \%{ }^{(5)}$.

Se concluye que la hepatitis A aguda es causa frecuente de síndrome febril en el norte del Perú, lo que muestra su importancia en la salud pública, principalmente en pacientes adolescentes o adultos de alto riesgo.

Fuentes de financiamiento: el Hospital Regional Lambayeque, a través de la estrategia sanitaria articulado nutricional, financió los reactivos, mientras que la Dirección de Investigación de la misma institución financió los gastos de uso de infraestructura y equipamiento de laboratorio.

Conflictos de interés: los autores declaran no tener conflicto de interés.

\section{REFERENCIAS BIBLIOGRÁFICAS}

1. Hernández R, Chaparro E, Díaz C, Carbajal M, Cieza É, Cerpa R. Frecuencia de hepatitis A en niños y adolescentes de cinco ciudades del Perú. Rev Peru Med Exp Salud Publica. 2015;32(3):499-503.

2. World Health Organization. Hepatitis A [Internet]. Ginebra: World Health Organization; 2015 [citado el 29 de noviembre de 2015]. Disponible en: http://www.who.int/mediacentre/ factsheets/fs328/es/

3. Aggarwal R, Goel A. Hepatitis A: epidemiology in resourcepoor countries. Curr Opin Infect Dis. 2015 Oct;28(5):48896. doi: 10.1097/QCO.0000000000000188.

4. Walker CM, Feng Z, Lemon SM. Reassessing immune control of hepatitis A virus. Curr Opin Virol. 2015 Apr;11:7-13. doi: 10.1016/j.coviro.2015.01.003

5. Daniels D, Grytdal S, Wasley A. Surveillance for acute viral hepatitis - United States, 2007. MMWR Surveill Summ. 2009 May;58(3):1-27.

Correspondencia: Heber Silva-Diaz

Dirección: Dirección de Investigación del Hospital Regional Lambayeque. Av. Via de Evitamiento Norte con Av. El Progreso. Chiclayo, Perú.

Correoelectrónico:hsilva@hrlamb.gob.pe

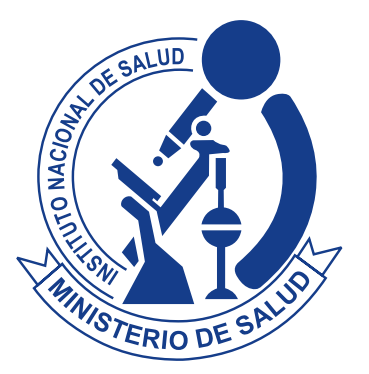

Investigar para proteger la salud
REVISTA PERUANA DE MEDICINA EXPERIMENTAL Y SALUD PÚBLICA CUMPLIENDO SUS METAS Y PROYECTÁNDOSE AL FUTURO

Visite los contenidos de la revista en: http://www.rpmesp.ins.gob.pe/ 